\title{
Compulsão alimentar e obesidade no climatério: uma revisão de literatura
}

\author{
Binge eating and obesity in climacteric: review of literature
}

Franciéli Aline Conte ${ }^{1}$, Lígia Beatriz Bento Franz¹, Vanessa Huber Idalêncio

`Universidade Regional do Noroeste do Estado do Rio Grande do Sul (UNIJUÍ) - ljuí (RS), Brasil.

DOI: http://dx.doi.org/10.7322/abcshs.v39i3.656

\section{RESUMO}

O climatério é uma fase de mudanças distintas na vida das mulheres caracterizada principalmente pelo hipoestrogenismo, por muitas mudanças fisiológicas e sintomas diversos. Devido à importante relevância desse tema à sociedade, buscou-se abordar a relação entre a compulsão alimentar e a obesidade no climatério. Foi realizada uma revisão de literatura por meio de busca científica de meio eletrônico e livros, a partir do ano de 2003, nas fontes de dados: Scientific Electronic Library Online (SciELO), Biblioteca Regional de Medicina (BIREME), Literatura Latino-Americana em Ciências da Saúde (LILACS) e Google Acadêmico. O sobrepeso e a obesidade são mais prevalentes no climatério, sendo a alimentação e o estilo de vida diretamente ligados a esses fatores. A ansiedade e o estresse podem dar origem a um problema muito comum e que repercute diretamente na alimentação e na não adesão às intervenções nutricionais: a compulsão por alimentos, conhecida como transtorno da compulsão alimentar periódica (TCAP). O climatério provoca o aumento da ansiedade e labilidade emocional pelo hipoestrogenismo, e o alívio da ansiedade ou estresse se dá, na maioria das vezes, pelo consumo de comida, o que favorece a obesidade e o aparecimento do TCAP.

Palavras-chave: climatério; obesidade; transtorno da compulsão alimentar.

\section{ABSTRACT}

Climacteric is a phase of relevant changings in the whole women's lives, especially characterized by hypoestrogenism and another physiological changes and multiple symptoms. Due to the relevance of this important topic to the entire society, we sought to address the relationship between binge eating and obesity in menopause. This paper is based on a review through scientific literature as electronic media and books from the year 2003, using the sources of data: Scientific Electronic Library Online (SciELO), Biblioteca Regional de Medicina (BIREME), Literatura Latino-Americana em Ciências da Saúde (LILACS) and Google Scholar. Overweight and obesity are more prevalent in the climacteric, with food intake and lifestyle directly connected to these factors. Anxiety and stress can lead to a very common problem that impacts directly on food and non-adherence to nutritional interventions: the food cravings, known as binge eating disorder (BED). The menopause can cause increased anxiety and emotional lability because of hipoestrogenism and the alleviation of anxiety or stress often comes through the food, favoring the onset of obesity and BED at this stage.

Keywords: climacteric; obesity; binge-eating disorder. 


\section{INTRODUÇÃO}

O período do climatério é uma fase natural para as mulheres que pode se iniciar aos 35 anos e prolongar-se até os 65. Esse período apresenta grande relevância em suas vidas, visto que ocorrem alguns fenômenos ao logo da fase reprodutiva que perpetuam com o aumento da idade, como o próprio envelhecimento, as mudanças na composição corporal, na pele, no metabolismo, entre outros ${ }^{1}$.

De acordo com a Organização Mundial da Saúde (OMS), o climatério pode ser entendido como uma "fase biológica da vida da mulher que compreende a transição entre o período reprodutivo e o não reprodutivo". Esse período pode ser dividido em três fases principais: pré-menopausa, perimenopausa e pós-menopausa. Cada uma delas possui características específicas e distintas formas de sintomas, que variam de mulher para mulher, mas que são muito comuns à maioria delas. Sintomas como fogachos, sudorese, irritabilidade, insônia, ganho ponderal e aumento da gordura na região abdominal são os mais comuns ${ }^{2}$.

Modificações do estado emocional no climatério são comuns e se dão principalmente pelo hipoestrogenismo, visto que a redução hormonal interfere na síntese dos neurotransmissores, o qual modula o comportamento e provoca alterações psíquicas, como mudanças comportamentais, labilidade emocional, maior dificuldade com a memória. Durante o climatério, outra mudança perceptível é o ganho ponderal (cerca de 0,8 kg/ano); após a menopausa, a gordura corporal pode aumentar cerca de $20 \%$. Para este evento, a principal causa é o descontrole ou a inadequação alimentar ${ }^{3}$.

As mudanças de comportamento alimentar são cada vez mais frequentes; as alterações no perfil alimentar atual, a grande oferta de alimentos, a idade, o grau de escolaridade, o contexto emocional para com os alimentos e o modelo de "beleza" imposto pela sociedade são grandes fatores interferentes da composição corporal na atualidade ${ }^{4}$.

A ocorrência de sobrepeso e obesidade na população mundial leva em consideração fatores mútuos, como a questão genética, o sedentarismo, o aumento da idade, e fatores psicossociais. Percebe-se, cada vez mais, que as pessoas têm menos tempo, mais problemas, mais fatores estressantes, e uma das formas de minimizar as angústias se dá por intermédio da alimentação, principalmente por parte dos indivíduos obesos, já que a alimentação "acalma" e a sua falta ou redução causa irritabilidade 5 .

A compulsão alimentar é reconhecida por provocar episódios de descontrole da alimentação, ocasionando sentimentos de culpa e autorreprovação. Os transtornos de compulsão alimentar periódica (TCAPs) afetam em maior proporção as mulheres com idades entre 30 e 50 anos e são mais prevalentes na população obesa. Mediante o exposto, é perceptível identificar coincidência entre faixa de idade, climatério e compulsão ${ }^{6}$.

O TCAP é caracterizado, segundo Galvão, Claudino e Borges ${ }^{7}$, pela associação de alguns fatores como: comer rapidamente, comer até sentir-se cheio(a), comer grandes quantidades sem sentir fome, sentir repulsa por si mesmo(a), sentir depressão ou culpa após o momento da compulsão ${ }^{7}$. Os transtornos alimentares (TA) têm influência direta sobre o metabolismo, visto que desregulam vias de absorção, podendo descontrolar a glândula tireoide, assim como podem influenciar nos padrões de fome e saciedade, na compreensão de fome e vontade de comer, contribuindo, dessa forma, para as alterações clínicas e nutricionais, como a desregulação do metabolismo ou os transtornos da alimentação ${ }^{8}$.

A ansiedade é muito comum e é um fator que predispõe a maioria das pessoas ao ato de comer. No entanto, o ato de comer não está relacionado apenas com fatores de ansiedade ou com fatores biológicos mas também com a convivência em sociedade, a cultura local, as lembranças relacionadas com os alimentos, a busca pelo prazer por meio da alimentação'

O TCAP está associado a psicopatologias, ou seja, distúrbios psicológicos que podem ser caracterizados como estado depressivo, transtornos de imagem corporal, altos níveis de estresse, estado de obesidade, labilidade emocional (sobrecargas de emoção), sintomas de um desequilíbrio que afeta intimamente a alimentação em si, causando a compulsão alimentar mais acentuadamente em mulheres ${ }^{10}$.

O controle de regulação da fome e da saciedade se dá por meio de diversos mecanismos intrínsecos, porém, para o caso da compulsão, essa percepção torna-se comprometida. Hormônios como insulina e leptina, neuropeptídios intestinais, a colecistoquinina (CCK), peptídeo pancreático (PP) e a própria distensão da parede do estômago são alguns dos exemplos que fazem parte da regulação da fome e da saciedade. No caso de uma "crise", a pessoa passará a comer mesmo sem estar com fome, e cessará a ingestão a partir do momento que sentir algum desconforto, dor ou culpa ${ }^{6}$.

Possivelmente, uma das grandes causas do sobrepeso e obesidade no climatério está relacionada com as mudanças hormonais e psicológicas que surgem nesse período, situações que, por sua vez, acarretam ansiedade, nervosismo, ingestão alimentar descontrolada e uma possível compulsão por alimentos, acentuando a obesidade. Com base nessa perspectiva, este estudo objetivou abordar a complexa relação entre a compulsão alimentar a obesidade no climatério.

\section{MÉTODOS}

A pesquisa se deu na forma de revisão bibliográfica, por meio de fontes de pesquisa eletrônica nas bases de dados Scientific Electronic Library Online (SciELO), Biblioteca Regional de Medicina (BIREME), Literatura Latino-Americana em Ciências da Saúde (LILACS) e em livros sobre o tema. A seleção dos referenciais se deu por critério de ano: a partir de 2003. Foram selecionados 36 artigos publicados em português e sete em língua inglesa, tanto de revisão observacional como de intervenção, além de cinco monografias e dois trabalhos de conclusão de curso (TCCs). Além disso, foram utilizados quatro livros, sendo três impressos e um eletrônico, materiais do Ministério da Saúde do Brasil. A seleção dos artigos se deu de acordo com a relevância do tema, e os textos envolveram os seguintes assuntos: envelhecimento feminino, climatério, ansiedade, ganho ponderal e TCAP. 
Do total de documentos encontrados (47 englobando artigos, monografias, TCCs, livros), foram lidos todos os títulos e resumos de cada um, totalizando 41 artigos, dos quais foram excluídos 13 por não atingirem a expectativa esperada dentro do tema. Foram lidos na íntegra 31 artigos; destes, foram utilizados 18 para a concepção deste trabalho, além de três livros e duas monografias.

Mediante a consulta aos Descritores em Ciências da Saúde (DeCS), foram encontrados os seguintes termos: climatério; obesidade; transtorno da compulsão alimentar. Palavras não encontradas foram substituídas por termos próximos ou mesmo substantivos, tais como: envelhecimento feminino foi substituído por mulheres e envelhecimento; doenças crônicas substituíram doenças crônicas não transmissíveis; o termo envelhecimento foi encontrado a partir da palavra senescência.

Foram excluídos artigos que não contemplaram o tema esperado ou proposto. Artigos ou documentos que não abordavam os temas compulsão alimentar, mulheres, climatério, ansiedade, obesidade e alimentação também foram descartados.

\section{RESULTADOS}

O climatério possui a ação multifatorial que corrobora muitos sintomas tidos como desconfortáveis e interferentes na qualidade de vida. Esse período é demarcado por hipoestrogenismo, esgotamento dos folículos ovarianos, queda do estradiol e consequente interrupção dos ciclos menstruais, que serão responsáveis pela sintomatologia descrita, e também fatores psicossociais, socioculturais, psicológicos e do próprio envelhecimento ${ }^{11}$.

O sobrepeso e a obesidade são mais prevalentes na fase climatérica mediante a redução do estrogênio e os seus efeitos sobre a redução do metabolismo e o aumento da ansiedade, como já mencionado; entretanto, o ganho de peso, de modo genérico, é visto de forma estética e poucas vezes relacionado com fatores de risco aumentados para a ocorrência de problemas cardíacos, hipertensão arterial, dislipidemias, certos tipos de câncer, diabetes e outras patologias, como de fato é cientificamente comprovado ${ }^{12}$.

A obesidade é algo expresso e visível; ao contrário das patologias citadas, acarreta aos portadores problemas com a autoestima, aceitação de si mesmo(a), desejo de ser magro(a), além da maior propensão a distúrbios relacionados à alimentação, especialmente entre as mulheres ${ }^{13}$.

A busca pela diminuição do peso corporal pode ser frustrante quando não se consegue atingir o objetivo. As inúmeras tentativas dão espaço, por exemplo, à desistência e a não adesão aos tratamentos nutricionais, pois não se trata apenas da questão fisiológica das pessoas, mas sim de um conjunto de fatores associados principalmente aos problemas psicoculturais ${ }^{14}$.

A mulher possui maior vulnerabilidade do que o homem em relação aos sintomas de ansiedade e depressão, e estes estão associados ao período reprodutivo. Estudos realizados em serviços de ginecologia identificaram maior prevalência de sintomas depressivos durante a perimenopausa e a menopausa, períodos marcados por irritabilidade, labilidade emocional, episódios frequentes de choro, ansiedade, entre outros sintomas ${ }^{15}$.

De acordo com Claudino e Zanella ${ }^{6}$, a solidão, o tédio, a raiva, a ansiedade e o estresse são os principais fatores que favorecem o desenvolvimento de TCAP. Para que seja possível realizar tal diagnóstico pela área da nutrição, aplica-se o diário alimentar. Para o tratamento da compulsão, é necessária ação multidisciplinar com o auxílio de clínico geral, psicólogos, psiquiatras e nutricionistas.

Além de todos os sintomas presentes na fase do climatério, o sobrepeso e a obesidade são atenuados a partir dessa fase, e tais fatores devem ser investigados a fim de se conhecer as possíveis relações entre os distúrbios alimentares e os agravos relacionados à saúde dessas mulheres ${ }^{16}$. Sob essa óptica, afirma-se que, entre outras causas, o ganho ponderal, além de estar envolvido com os fatores genéticos, o climatério e o próprio envelhecimento, é também consequência de fatores associados à ansiedade, às alterações de humor e às preocupações, situações que deixam as pessoas mais vulneráveis e buscando "acalmar-se" por meio da ingestão de alimentos ${ }^{17}$.

\section{DISCUSSÃO}

A obesidade tem se agravado no mundo todo. No Brasil, a prevalência de sobrepeso, no ano de 2012, foi de 55,9\% para mulheres entre 45 e 54 anos de idade, e $60,9 \%$ para mulheres entre 55 e 64 anos. A prevalência de obesidade foi constatada em maior proporção em mulheres na faixa entre 55 e 64 anos (24,5\%), enquanto para os homens dessa idade a prevalência foi de $15,7 \%$. Verificou-se que $23,9 \%$ das mulheres com idade entre 45 e 54 anos eram obesas, enquanto $19,4 \%$ dos homens na mesma faixa etária foram classificados como obesos. Verifica-se, portanto, que o sobrepeso e a obesidade prevalentes nas mulheres correspondem à faixa relacionada ao climatério ${ }^{18}$.

Um estudo realizado com 113 mulheres com idade entre 22 e 60 anos $(39,35 \pm 10,85)$ participantes de programas para redução de peso em Porto Alegre (RS) demonstrou, mediante entrevistas estruturadas, Inventário de Ansiedade de Beck (BAI), Inventário de Depressão de Beck (BDI) e Escala de Compulsão Alimentar Periódica (ECAP), que a compulsão alimentar possui associação positiva com os sintomas de ansiedade e depressão, porém não possui associação com o índice de massa corpórea (IMC) ${ }^{19}$.

O climatério pode ocasionar inúmeras modificações no organismo, desencadeando sintomas como instabilidade emocional e ansiedade. A ansiedade se caracteriza por provocar irritabilidade, sudorese, fadiga, taquicardia, inquietude, cansaço, dificuldade de concentração, tensão muscular e insônia ${ }^{18}$. Um estudo realizado com 749 mulheres no climatério demonstrou que a ansiedade esteve presente em $49,8 \%$ das estudadas, e que os principais fatores associados são os hábitos de vida e as condições socioeconômicas das participantes ${ }^{20}$.

A ansiedade pode desencadear muitos sintomas e comportamentos, tais como: sudorese, inquietações, taquicardia. Para o 
controle de tal característica, muitas pessoas buscam "se aliviar" e "descarregar" a tensão e a irritabilidade por meio de algo que lhes proporcione prazer ou aquietação. Uma das formas se dá mediante a comida, o que favorece a epidemia de obesidade. A continuidade desse processo de ansiedade e alívio ao se alimentar torna-se um círculo vicioso que pode levar posteriormente ao TCAP ${ }^{21}$.

O controle da compulsão deve se dar de forma interdisciplinar e grupal, visto que reuniões motivam os indivíduos a mudarem o seu comportamento alimentar, pois eles socializam os seus problemas e ao mesmo tempo aumentam o seu conhecimento acerca do problema enfrentado ${ }^{14}$, ou seja: ele(a) não está sozinho(a) e conta com o apoio de pessoas que vivem um problema em comum e com uma equipe que trabalha o sintoma da compulsão e a melhora dos hábitos alimentares ${ }^{22}$.

Ao focar a obesidade e o climatério, é possível perceber uma íntima associação entre estes e ansiedade, labilidade emocional e TCAP. Levando-se em conta que TA são complexos, verifica-se a necessidade de diagnóstico e tratamento de forma interdisciplinar (entre profissionais da psicologia e da nutrição).

O diagnóstico e o controle nutricional podem ser realizados mediante a aplicação de diário alimentar, o qual auxiliará no automonitoramento. Além disso, constata-se a necessidade da aplicação da anamnese alimentar, em que o exame deve abranger informações sobre atividade física, história da doença e situação atual, hábitos alimentares por meio de um recordatório de 24 horas (quantidade, frequência e modo de preparo), além de dados que levem em consideração a ingestão hídrica e o funcionamento intestinal (consistência das fezes, número de evacuações, horários), e um trabalho voltado à questão da saciedade, seleção de alimentos e da fome em sí23.

Diagnosticar a compulsão no referido ciclo torna-se uma grande ferramenta de apoio para o controle/eliminação das crises. Desse modo, os tratamentos nutricional e psíquico, desde que sejam contínuos, tornam-se fundamentais para a adesão aos planos alimentares, aconselhamentos dietéticos e do controle da ansiedade e da vontade de comer compulsivamente, auxiliando, dessa forma, na redução do peso da massa corporal e dos riscos à saúde desencadeados pela obesidade e má alimentação.

O climatério não pode ser visto apenas como uma fase "problemática" ou desencadeadora de sintomas negativos, por ocasionar mudanças corporais e psicológicas, mas sim como uma etapa que se dá de forma individualizada e em menor ou maior proporção; as individualidades e as realidades de cada mulher devem ser consideradas ${ }^{24}$.

Pelos sintomas característicos do climatério, é muito comum associar este a um evento estritamente negativo. No entanto, o climatério é também o período em que a grande maioria das mulheres tem maturidade para realizar seus objetivos, possuir estabilidade, por exemplo. Os efeitos do climatério podem ser reduzidos/amenizados por meio da adesão a estilos de vida saudáveis, mediante a procura de recursos (profissionais da saúde) quando constatados desconfortos desse período.

\section{CONCLUSÃO}

O climatério provoca aumento da ansiedade e labilidade emocional pelo hipoestrogenismo, e o alívio da ansiedade ou estresse se dá, na maioria das vezes, pelo consumo de comida, o que favorece a obesidade e o aparecimento do TCAP nessa fase. $\mathrm{O}$ tratamento mais indicado para esse período se dá por meio da terapia dietética e de acompanhamento psicológico, a fim de auxiliar na perda ponderal e na melhora dos sintomas de ansiedade e descontrole da alimentação. A psicologia e a nutrição têm função imprescindível no tratamento da compulsão. A nutrição exerce um papel relevante para a adequação calórica e nutricional e de educação alimentar nesse período.

\section{REFERÊNCIAS}

1. Gallon CW, Wender MCO. Estado nutricional e qualidade de vida da mulher climatérica. Rev Bras Ginecol Obstet. 2012;34(4):175-83. http://dx.doi.org/10.1590/S0100-72032012000400007

2. Pedro AO, Pinto-Neto AM, Costa-Paiva LHS, Osis MJD, Hardy EE. Síndrome do climatério: inquérito populacional domiciliar em Campinas, SP. Rev Saúde Pública. 2003;37(6):735-42. http://dx.doi.org/10.1590/S0034-89102003000600008

3. Lorenzi DRS, Catan LB, Moreira K, Ártico GR. Assistência à mulher climatérica:novosparadigmas. RevBrasEnferm. 2009;62(2):287-93. http://dx.doi.org/10.1590/S0034-71672009000200019

4. Boris GDJB. Mulher, corpo e subjetividade: uma análise desde - patriarcado à contemporaneidade. Rev Mal-Estar Subj. 2007;6(2):451-78.
5. Fleury HJ, Marra MM. Intervenções grupais na saúde. São Paulo: Ágora; 2005.

6. Claudino AM, Zanella MT. Guia de transtornos alimentares e obesidade. São Paulo: Manole; 2005. p. 332.

7. Galvão AL, Claudino AM, Borges MBF. Aspectos históricos e evolução do diagnóstico. In: Nunes MA. Transtornos alimentares e obesidade. 2 ed. São Paulo: Artmed; 2006. p. 45

8. Barreto AL, Figueiró AS, Soares RM. Padrão alimentar e manejo nutricional dos transtornos alimentares. In: Nunes MA, Appolinário JC, Galvão AL, Coutinho W. Transtornos alimentares e obesidade. 2 ed. São Paulo: Artmed; 2006. p.117.

9. Passos TCBM, Yazigi L, Claudino AM. Aspectos ideativos no transtorno da compulsão alimentar periódica: estudo com o Rorschach. Psico-USF. 2008:13(1):69-74 http://dx.doi.org/10.1590/S1413-82712008000100009 
10. Romano ECB, Philippi ST. O que é compulsão alimentar? O que é episódio bulímico? In: Philippi ST, Alvarenga M. Transtornos alimentares: uma visão nutricional. Barueri: Manole; 2004. p. 194.

11. De Lorenzi DRS, Danelon C, Saciloto B, Padilha Junior I. Fatores indicadores da sintomatologia climatérica. Rev Bras Ginecol Obstet. 2005;27(1):7-11.

http://dx.doi.org/10.1590/S0100-72032005000100004

12. Lessa M. Doenças ligadas à obesidade custam $R \$ 488$ milhões [Internet]. Disponível em: http://sna.saude.gov.br/noticias. cfm?id=5013. Acesso em: 24 abr. 2014.

13. Almeida CME, Oliveira MRM, Vieira CM. A relação entre a imagem corporal e obesidade em usuárias de Unidades de Saúde da Família. Rev Simbio-Logias. 2008;1(1):111-21.

14. Vieira CM, Turato ER. Percepções de pacientes sobre alimentação no seu processo de adoecimento crônico por síndrome metabólica: um estudo qualitativo. Rev Nutrição. 2010;23(3):425-32. http://dx.doi.org/10.1590/S1415-52732010000300010

15. Andrade LHSG, Viana MC, Silveira CM. Epidemiologia dos transtornos psiquiátricos na mulher. Rev Psiq Clín. 2006;33(2)43-54.

16. Azevedo AP, Santos CC, Fonseca DC. Transtorno da compulsão alimentar periódica. Rev Psiq Clín. 2004;31(4):170-2.

17. Montilla RNG, Marucci MFN, Aldrighi JM. Avaliação do estado nutricional e do consumo alimentar de mulheres no climatério. Rev Assoc Med Bras. 2003;49(1):91-5. http://dx.doi.org/10.1590/S0104-42302003000100040
18. Brasil. Ministério da Saúde. Secretaria de Vigilância em Saúde. Secretaria de Gestão Estratégica e Participativa. Vigitel Brasil 2011: Vigilância de fatores de risco e proteção para doenças crônicas por inquérito telefônico. Brasília: Ministério da Saúde; 2012. p. 132.

19. Bittencourt SA, Lucena-Santos P, Moraes JFD, Oliveira MS. Anxiety and depression symptoms in women with and without binge eating disorder enrolled in weight loss programs. Trends Psychiatry Psychother. 2012;34(2):87-92. http://dx.doi.org/10.1590/S2237-60892012000200007

20. Cortez CM, Araújo EA, Ribeiro MV. Transtorno de compulsão alimentar periódico e obesidade. Arq Catarin Med. 2011;40(1):94-102.

21. Associação Brasileira de Psiquiatria. Transtornos de Ansiedade: diagnóstico e tratamento. Projeto Diretrizes; 2008 [internet]. Disponível em: http://www.projetodiretrizes.org.br/projeto_ diretrizes/099.pdf. Acesso em: 24 Abr. 2014.

22. Pereira WMP, Schmitt ACB, Buchalla CM, Reis AOA, Aldrighi JM Ansiedade no climatério: prevalência e fatores associados. Rev Bras Crescimento Desenvolvimento Hum. 2009;19(1):89-97.

23. Sarubbi EB. Uma abordagem de tratamento psicológico para a compulsão alimentar. Dissertação (Mestrado) - Universidade Católica Dom Bosco. Campo Grande: 2003, p.83.

24. Conte FA, Franz LBB, Weber ITSW, Steinmetz SB, Buss V, Manenti M. A compulsão alimentar no climatério: um artigo de revisão. Salão do conhecimento; ljuí, 2013. 